\title{
BMJ Open Multicentre, randomised clinical trial comparing intravitreal aflibercept monotherapy versus aflibercept combined with reduced-fluence photodynamic therapy (RF-PDT) for the treatment of polypoidal choroidal vasculopathy
}

Chinmayi Himanshuroy Vyas, ${ }^{1,2}$ Chui Ming Gemmy Cheung (1) , ${ }^{1,2}$ Colin Tan, ${ }^{3}$ Caroline Chee, ${ }^{4}$ Kelly Wong, ${ }^{1}$ Janice Marie N. Jordan-Yu, ${ }^{1}$ Tien Yin Wong (1) , ${ }^{1,2}$ Anna Tan, ${ }^{1,2}$ Beau Fenner, ${ }^{2}$ Shaun Sim, ${ }^{2}$ Kelvin Yi Chong Teo (D) ${ }^{1,2}$

To cite: Vyas $\mathrm{CH}$, Cheung $\mathrm{CMG}$, Tan C, et al. Multicentre, randomised clinical trial comparing intravitreal aflibercept monotherapy versus aflibercept combined with reduced-fluence photodynamic therapy (RF-PDT) for the treatment of polypoidal choroidal vasculopathy. BMJ Open 2021;11:e050252. doi:10.1136/ bmjopen-2021-050252

- Prepublication history and additional supplemental material for this paper are available online. To view these files, please visit the journal online. To view these files, please visit the journal online (http://dx.doi. org/10.1136/bmjopen-2021050252).

Received 18 February 2021 Accepted 18 June 2021

D) Check for updates

(c) Author(s) (or their employer(s)) 2021. Re-use permitted under CC BY-NC. No commercial re-use. See rights and permissions. Published by BMJ.

For numbered affiliations see end of article.

\section{Correspondence to} Dr Kelvin Yi Chong Teo; kelvin.teo.y.c@singhealth. com.sg

\section{ABSTRACT}

Purpose To compare the efficacy and safety of intravitreal aflibercept (IVA) monotherapy versus aflibercept combined with reduced-fluence photodynamic therapy (RF-PDT) (IVA+RF-PDT) for the treatment of polypoidal choroidal vasculopathy (PCV).

Methods and analysis Multicentred, double-masked, randomised controlled trial to compare the two treatment modalities. The primary outcome of the study is to compare the 52-week visual outcome of IVA versus IVA+RF PDT. One hundred and sixty treatment-naïve patients with macular PCV confirmed on indocyanine green angiography will be recruited from three centres in Singapore. Eligible patients will be randomised (1:1 ratio) into one of the following groups: IVA monotherapy group-aflibercept monotherapy with sham photodynamic therapy $(n=80)$; combination group-aflibercept with RF-PDT $(n=80)$. Following baseline visit, all patients will be monitored at 4 weekly intervals during which disease activity will be assessed based on best-corrected visual acuity (BCVA), ophthalmic examination findings, optical coherence tomography (OCT) and angiography where indicated. Eyes that meet protocol-specified retreatment criteria will receive IVA and sham/RF-PDT according to their randomisation group. Primary endpoint will be assessed as change in BCVA at week 52 from baseline. Secondary endpoints will include anatomical changes based on OCT and dye angiography as well as safety assessment. Additionally, we will be collecting optical coherence tomography angiography data prospectively for exploratory analysis.

Ethics and dissemination This study will be conducted in accordance with the ethical principles that have their origin in the Declaration of Helsinki and that are consistent with the ICH E6 guidelines of Good Clinical Practice and the applicable regulatory requirements. Approval from the SingHealth Centralised Institutional Review Board has been sought prior to commencement of the study.

Trial registration number NCT03941587.
Strength and limitations of this study

Multicentre randomised clinical trial with masked image grading by an independent ophthalmology reading centre.

- First randomised clinical trial to compare the efficacy of intravitreal aflibercept (IVA) versus IVA+reducedfluence photodynamic therapy (RF-PDT) in an Asian population.

- Baseline RF-PDT to be formally evaluated in combination with aflibercept.

- Prospective optical coherence tomography angiography evaluation for analysis.

- Limitation: no head-on comparison between full fluence and RF-PDT.

\section{INTRODUCTION}

Polypoidal choroidal vasculopathy $(\mathrm{PCV})$ is a subtype of neovascular age-related macular degeneration (nAMD) which comprises up to $50 \%$ of exudative maculopathy in the Asian population. ${ }^{1-5}$ Antivascular endothelial growth factor (VEGF) therapy has been established as the standard of care for treatment of $\mathrm{nAMD}^{6-9}$; however, the response in eyes with PCV has been less consistent. ${ }^{10-13}$ Current best evidence for the treatment of PCV comes from two recent randomised controlled trials. The EVEREST II study reported superior visual gains and lower number of anti-VEGF treatments in the intravitreal ranibizumab combined with photodynamic therapy (IVR+PDT) arm compared with IVR monotherapy. ${ }^{1415}$ On the other hand, the PLANET study reported good visual gain (>10 letters) in the intravitreal aflibercept (IVA) monotherapy arm and demonstrated 
no additional benefit of IVA with rescue photodynamic therapy (PDT) ${ }^{16}{ }^{17}$ Hence, both approaches are currently practised. However, there remains a gap in the evidence which is specific to aflibercept. Despite the impressive visual gain with IVA monotherapy, limited polypoidal lesion (PL) closure rate was achieved with IVA monotherapy. The lower rate of PL closure without the use of PDT in combination with anti-VEGF therapy remains an area of concern clinically as unclosed PL may require regular long-term treatment or increase risk of recurrence and haemorrhage. ${ }^{18}$ There have been no randomised controlled trials to date to evaluate whether addition of PDT to IVA would result in superior visual and/or PL closure compared with IVA monotherapy.

Here we report the methodology of a study designed to evaluate the efficacy and safety of combination IVA and PDT in patients with PCV. We hypothesise that the combination group will achieve comparable visual outcomes and higher PL closure rate with lower number of retreatments compared with IVA monotherapy. In addition, this study will use reduced-fluence photodynamic therapy (RF-PDT), which has been reported to have a better safety profile compared with full-fluence PDT. ${ }^{19}{ }^{20}$ Finally, this protocol will incorporate the analysis of novel imaging biomarkers, such as choroidal features like choroidal vascular hyperpermeability $(\mathrm{CVH})^{21} 22$ and Choroidal Vascularity Index (CVI) ${ }^{23}$ Specifically, optical coherence tomography angiography (OCTA) will be performed during every study visit, thus allowing for longitudinal analysis within a RCT setting.

\section{METHODS AND ANALYSIS}

\section{Study type and study design}

Multicentred randomised controlled trial registered with Clinical trial.gov (http://clinicaltrials.gov/show/). The trial registration dataset in accordance with WHO is summarised in online supplemental table 1 (protocol number: R1735/58/2020, protocol V.1.1/02, October 2020).

\section{Study title}

A multicentre, randomised clinical trial comparing IVA monotherapy versus aflibercept combined with RF-PDT for the treatment of PCV.

\section{Principal investigator}

Professor Gemmy Cheung Chui Ming, FRCSOphth

Singapore Eye Research Institute

\section{Study setting}

Site 1: Singapore National Eye Centre (SNEC).

Contact: Professor Gemmy Cheung Chui Ming.

Site 2: National Healthcare Group Eye Institute, Tan Tock Seng Hospital (TTSH), Singapore.

Contact: A/Professor Colin Tan.

Site 3: National University Hospital (NUH), Singapore. Contact: A/Professor Caroline Chee.
Central reading centre

Singapore National Eye Centre Ocular Reading Centre (SORC).

\section{Study outcomes}

Primary outcomes

The primary outcome of this study is to compare the change in BCVA from baseline to week 52 between the monotherapy group (IVA) and the combination group (IVA+RF-PDT).

The primary visual outcomes are

- Final BCVA at week 52.

- Proportion of eyes with gain $\geq 5,10,15$ letters at week 52.

- Proportion of eyes with loss $\geq 5,10,15$ letters at week 52.

- Proportion of eyes with 70 or more logMAR letters.

- Comparison of secondary visual outcomes between groups.

\section{Secondary outcomes}

The secondary anatomical outcomes are

To compare anatomical outcomes at weeks 12 and 52 between treatment groups assessed by multimodal imaging

- Optical coherence tomography (OCT): for evidence of intraretinal or subretinal fluid, ill-defined hyperreflective material and/or new haemorrhage (time frame: every month for 12 months).

- OCT-angiograph (OCT-A): for evidence of intraretinal or subretinal fluid, ill-defined hyper-reflective material and/or new haemorrhage (time frame: every month for 12 months).

- Colour fundus photography: to inspect anomalies associated with diseases that affect the eye and to monitor their progression (time frame: baseline, month 3 and month 12).

- Autofluorescence photography retinal imaging (time frame: baseline, month 3 and month 12).

- Fundus fluorescein angiography for retinal circulation (time frame: baseline, month 3 and month 12).

- Intraocular pressure (IOP) fluid pressure in the eye (time frame: baseline and 12 months).

The secondary management outcomes are

- Total number of intravitreal injection of aflibercept and RF-PDT treatment.

- Retreatment number of intravitreal injection of aflibercept and RF-PDT treatment.

\section{Exploratory imaging analysis}

Imaging predictors CVI, CVH and sub-RPE hyperreflective ring.

Longitudinal changes in OCTA between the groups.

\section{Outcome definitions}

Visual assessment of BCVA will be determined according to the $\log$ MAR letters in all visits. 
PL closure will be defined as a PL disappearance as assessed by investigators and reading centre on indocyanine green angiography (ICGA).

\section{Efficacy assessment}

All efficacy assessments include both functional and anatomical evaluations. These include comparisons of the following between the groups: BCVA, PL closure on ICGA, presence of intralesional or sublesional fluid on SD-OCT and OCTA.

\section{Safety assessment}

Safety parameter will include assessment of IOP, adverse events (AEs) and serious adverse events (SAEs).

\section{Timeline}

A total of 160 patients with PCV recruited over approximately 16 months' period (starting February 2021) total follow-up time of 52 weeks after inclusion.

Study start date : 1 February 2021.

Anticipated study end date : 31 May 2022.

\section{Sample selection}

The study population will consist of a group of adults aged 50 and above with symptomatic macular PCV who are naive to treatment in the study eye. Study participants can only have one study eye. If both eyes are eligible for the study, the eye without previous intravitreal anti-VEGF treatment will be selected. If both eyes are treatment naïve, the eye with worse BCVA should be selected as the study eye. There will be no restriction of recruitment according to the race of the patient.

\section{Eligibility criteria}

Potential eligibility will be assessed as part of a routinecare examination. Prior to completing any procedures or collecting any data that are not part of usual care, written informed consent will be obtained. For potential study participants who are considered potentially eligible for the study based on a routine-care exam, the study protocol will be discussed with the potential study participant by a study investigator and study coordinator. The potential study participant will be given the informed consent form to read. Potential study participants will be encouraged to discuss the study with family members and their personal physician(s) before deciding whether to participate in the study.

\section{Inclusion criteria}

- Male or female study participant aged $\geq 50$ years old at the time of informed consent.

- BCVA early treatment diabetic retinopathy study (ETDRS) letters score of 4-73 letters (Snellen equivalent approximately 20/32 to 20/800) in the study eye.

- Confirmed diagnosis of symptomatic macular PCVbased ICGA with activity of PCV confirmed by exudation involving the macula on OCT or FA or both.

- Presence of intraretinal or subretinal fluid/blood at the fovea as seen on OCT.
- Treatment naïve.

- *No previous treatment with intravitreal anti-VEGF agents, regardless of the indication.

- *No previous thermal laser in the macular region, or verteporfin PDT, regardless of indication.

- *No other previous treatment for nAMD, except oral supplements and traditional Chinese medicine.

- Greatest linear dimension of the total lesion area branching vascular network (BVN) $+\mathrm{PL}<5400 \mu \mathrm{m}(\sim 9$ MPS disc areas) as delineated by ICGA.

- Able and willing to provide written informed consent and comply with all scheduled visits and study procedure.

\section{Exclusion criteria}

Participant

- Medical condition that, in the opinion of the investigator, would preclude participation in the study (eg, unstable medical status including blood pressure, cardiovascular disease and glycaemic control).

- Participation in an investigational trial within 30 days of enrolment which involves treatment with unapproved investigational drug.

- Known allergy to any component of the study drug.

- Blood pressure of $>180 / 110$ (systolic above 180 or diastolic above 110 on repeated measurements). If blood pressure is brought below $180 / 110$ by antihypertensive treatment, individual can become eligible.

- Myocardial infarction, other acute cardiac event requiring hospitalisation, stroke, transient ischaemic attack or treatment for acute congestive heart failure within 4 months prior to randomisation.

- Systemic anti-VEGF or pro-VEGF treatment within 4 months prior to randomisation or anticipated use during the study.

- Severe amblyopia $($ BCVA $<20 / 100)$ or blind in fellow eye $(\mathrm{BCVA}<20 / 800)$.

\section{Study eye}

- Eye with intraretinal or subretinal fluid due to other causes than PCV.

- An ocular condition is present (other than PCV) that, in the opinion of the investigator, might affect intraretinal or subretinal fluid or alter visual acuity during the course of the study (eg, DME, vein occlusion, uveitis or other ocular inflammatory disease, neovascular glaucoma, etc).

- Substantial cataract that, in the opinion of the investigator, is likely to be decreasing visual acuity by more than three lines (ie, cataract would be reducing acuity toworse than 20/40 if eye was otherwise normal).

- Any intraocular surgery within 3 months of enrolment.

- Treatment with intravitreal corticosteroids.

- History of retinal detachment or surgery for retinal detachment.

- History of vitrectomy.

- History of macular hole. 
- Evidence of vitreomacular traction that may preclude resolution of macular oedema of $>4$ disc areas of intraretinal/subretinal haemorrhage.

- Aphakia.

- Exam evidence ofexternal ocular infection, including conjunctivitis, chalazion or significant blepharitis.

\section{Other eye}

- Active intraocular inflammation.

- History of uveitis.

Patient withdrawal

Participants may voluntarily withdraw from the study at any time. If a study participant is considering withdrawal from the study, the principal investigator should personally speak to the individual about the reasons, and every effort should be made to accommodate him or her.

Study participants who withdraw will be asked to have a final closeout visit at which the testing described for the protocol visits will be performed. Study participants who have an adverse effect attributable to a study treatment or procedure will be asked to continue in follow-up until the AE has resolved or stabilised.

\section{Study assessment and visit schedule/study procedures/data acquisition \\ Screening evaluation and baseline testing Historical information}

A history will be elicited from the potential study participant and extracted from available medical records. Data to be collected will include age, gender, ethnicity and race, medical history and medications being used, as well as ocular diseases, surgeries and treatment.

\section{Baseline testing procedures}

The following procedures are needed to assess eligibility and/or to serve as baseline measures for the study:

- BCVA: BCVA will be measured using the ETDRS VA protocol following manifest refraction.
- SD-OCT: Spectralis OCT (Heidelberg Engineering, Heidelberg, Germany) and Topcon DRI OCT Triton/V.10.17.003.03 (Tokyo, Japan) will be used for image acquisition. Both standard and enhanced depth imaging scans will be performed.

- OCTA will be performed at baseline, and at each subsequent visits, Topcon DRI OCT Triton/V.10.17.003.03 will be used to perform the OCTA.

- Ocular examination on each eye including slit lamp, measurement of IOP, lens assessment and dilated fundus examination (within 21 days prior to randomisation).

- Blood pressure measurements (Dinamap 1846 XT; Critiko Corporation, Tempa, Florida, USA).

- Fundus photography will be performed on TRC-50X/ IMAGEnet 2000 (Topcon).

- Autofluorescence photography will be performed on the Spectralis OCT (Heidelberg Engineering).

- Fundus fluorescein and ICGA: FFA and ICGA will be performed (Spectralis OCT, Heidelberg Engineering).

Study participants will be randomised to two treatment groups using a ratio of 1:1 (combination group: aflibercept combined with RF-PDT, IVA; monotherapy group: aflibercept monotherapy with sham RF-PDT) (figure 1).

Disease characteristics of the study eye assessed by the investigator at screening (day 1):

- Diagnosis of PCV based on ICGA.

- PL presence will be defined as the presence of single or multiple hyperfluorescent lesions on ICGA within the first $6 \mathrm{~min}$ with one or more of the following features:

- Nodular appearance on stereoscopic view of ICGA.

- Hypofluorescent halo surrounding the focal hyperfluorescent lesion(s) on early frames.

- Pulsatile filling of the lesion on video ICGA.

- Presence of activity clinically as evidenced by presence of haemorrhage, oedema on fundus examination.

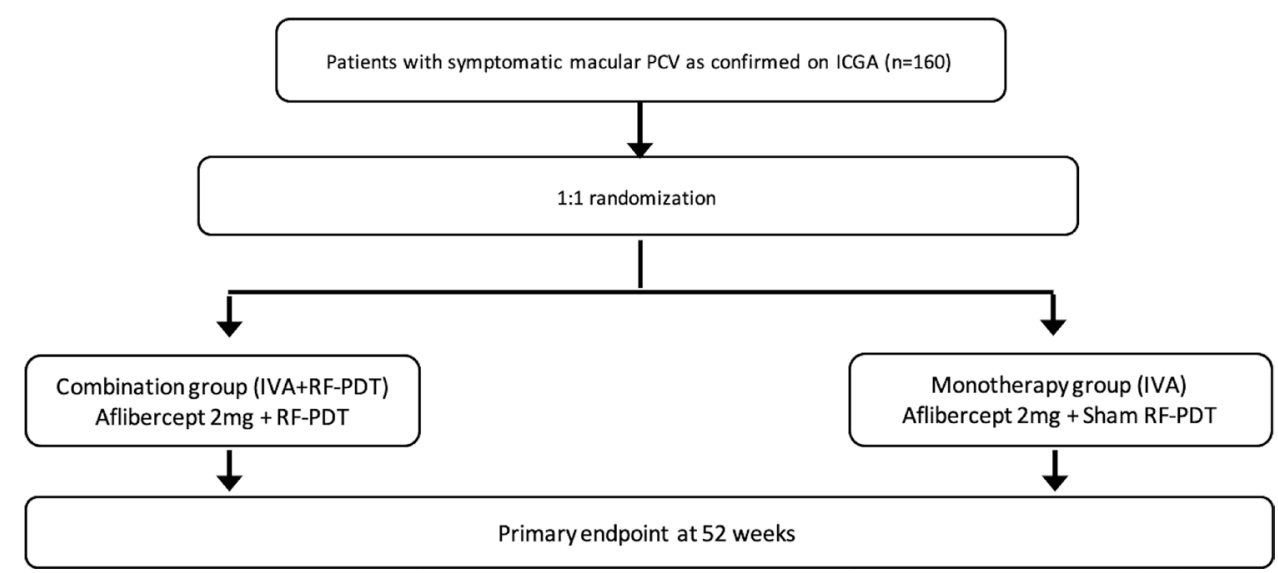

Figure 1 Randomisation of the patients in the monotherapy or combination therapy groups. Once enrolled, study participants will be randomised to two treatment groups using a ratio of 1:1: (1) combination group: aflibercept combined with RF-PDT, IVA; (2) monotherapy group: aflibercept monotherapy with sham RF-PDT. ICGA, indocyanine green angiography; IVA, intravitreal aflibercept; PCV, polypoidal choroidal vasculopathy; RF-PDT, reduced-fluence photodynamic therapy. 
- Presence of activity as evidenced by intraretinal or subretinal fluid on OCT.

\section{Follow-up visit schedule and examination}

At each visit, study participants will be assessed based on BCVA, ophthalmic examination, OCT and OCT-A. (table 1)

In addition, colour photography, autofluorescence photography, fluorescein and ICGA will be performed at baseline, week 12 and week 52 (table 1).

Additional investigations at interim visits may be performed at the discretion of the investigator if there is evidence of disease activity (figure 2).

Qualification for retreatment with aflibercept (figure 3) (weeks 4-48) will be based on signs of disease activity defined as persistent intraretinal or subretinal fluid on OCT and BCVA.

Qualification for retreatment with PDT (figure 3) (weeks 4-48, repeated not more frequently than 12 weeks apart) will be based on signs of persistent PL on ICGA, according to randomisation group.

\section{End of study visit}

At week 52, all subjects will return for end of study visit. All subjects will be assessed based on BCVA, ophthalmic examination, FA, ICGA, colour fundus photography, autofluorescence and OCT and OCT-A.

\section{Role of centralised reading centre}

All eligibility criteria and retreatment decisions will be based on investigators assessments. All images will be sent to the SORC at the conclusion of the clinical study for the purpose of analysis. These gradings will have no bearing on the retreatment decisions during the trial. The grading protocol is included in online supplemental table 2.

\section{Treatment procedure}

Study eyes will receive either intravitreal injection of aflibercept, along with sham RF-PDT or RF-PDT depending on the assigned randomisation group (figure 1).

When a patient's condition warrants treatment with both RF-PDT/sham RF-PDT and aflibercept, sham/ RF-PDT will be performed first.

\section{Intravitreal injection technique}

Antibiotics in the preinjection, peri-injection or postinjection period are not necessary but can be used at investigator discretion if such use is part of his/her usual routine.

Prior to the injection, the study eye will be anaesthetised with topical anaesthetic, followed by a povidone iodine prep of the conjunctiva. (Instil 5\% povidone iodide on to the ocular surface and allow adequate time prior to injection.)

Aflibercept will be withdrawn using aseptic technique through an 18-gauge filter needle attached to a $1 \mathrm{~mL}$ syringe. The needle will be discarded after withdrawal of the vial contents and should not be used for intravitreal injection. The needle should be replaced by a sterile 30-gauge needle for the intravitreal injections. The contents of the syringe should be expelled until the plunger is aligned with the line that marks $0.05 \mathrm{~mL}$ on the syringe.

The injection will be performed using sterile technique. The investigator will use a surgical hand disinfection technique and wear sterile gloves. Periocular skin and eyelid margins and eye lashes will be cleaned with $10 \%$ povidone iodine.

Skin will be dried and drape will be applied. The investigator will insert an eyelid speculum, ensuring that it is well positioned underneath the eyelids to direct the eyelashes away from the field. Callipers should be used to mark the injection site. The entry site of the needle should be $3.0-3.5 \mathrm{~mm}$ from the limbus in pseudophakic patients, and $3.5-4.0 \mathrm{~mm}$ in phakic patients.

The conjunctiva may be displaced anteriorly using either forceps or cotton tipped applicator so that no direct route between vitreous and ocular surface remains. The needle is inserted perpendicular through sclera with the tip aimed towards the centre of the globe (to avoid any contact with the posterior lens capsule)

IOP measurement postinjection is not mandatory. While small volume injections $(0.05 \mathrm{~mL})$ are unlikely to cause IOP rise, it should be considered in participants with ocular hypertension or glaucoma, and in all cases where participants are symptomatic for pain or reduced vision immediately following injection.

Should a high IOP resulting in non-perfusion of the central retinal artery occur, indicated by no perception of light in the treated eye, an anterior chamber paracentesis is indicated. Such decompression needs to be achieved within 3-5 min. Participants should be instructed to report any symptoms regarding eye pain or discomfort, increased redness of the eye or additional blurring of vision (which may indicate endophthalmitis) to the treating ophthalmologist without delay

\section{Delay in giving injections}

If a scheduled injection is not given on the day of study visit, it may be administered within 7 days after the occurrence of the study visit. If it is not given by that time, it will be considered missed. If an injection is given late, the next scheduled injection should occur no sooner than 28 days after the previous injection.

\section{Non-study eye injections}

If the non-study eye is going to be treated for any condition which requires treatment with an anti-VEGF agent, it may be treated at the discretion of the investigator. Treatment of the fellow- eye with aflibercept is possible.

\section{RF-PDT/sham RF-PDT administration}

Pretreatment patient preparation

The verteporfin (Visudyne) /sham (5\% dextrose in water solution for infusion) intravenous infusion will be administered using standard aseptic technique. The skin at the 


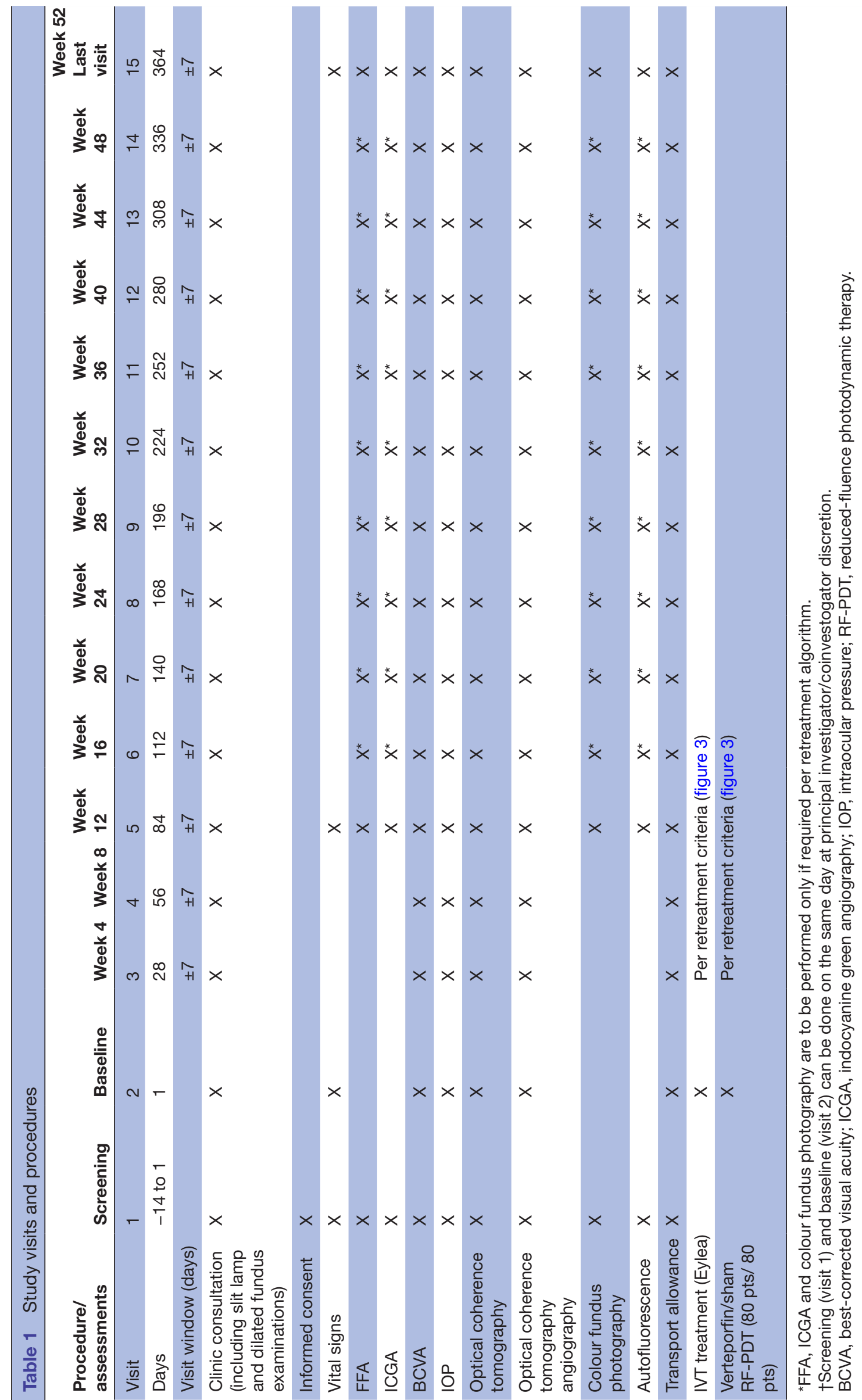

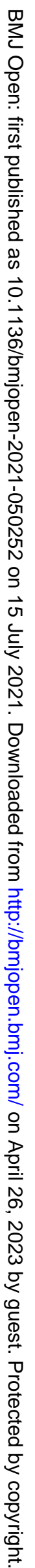




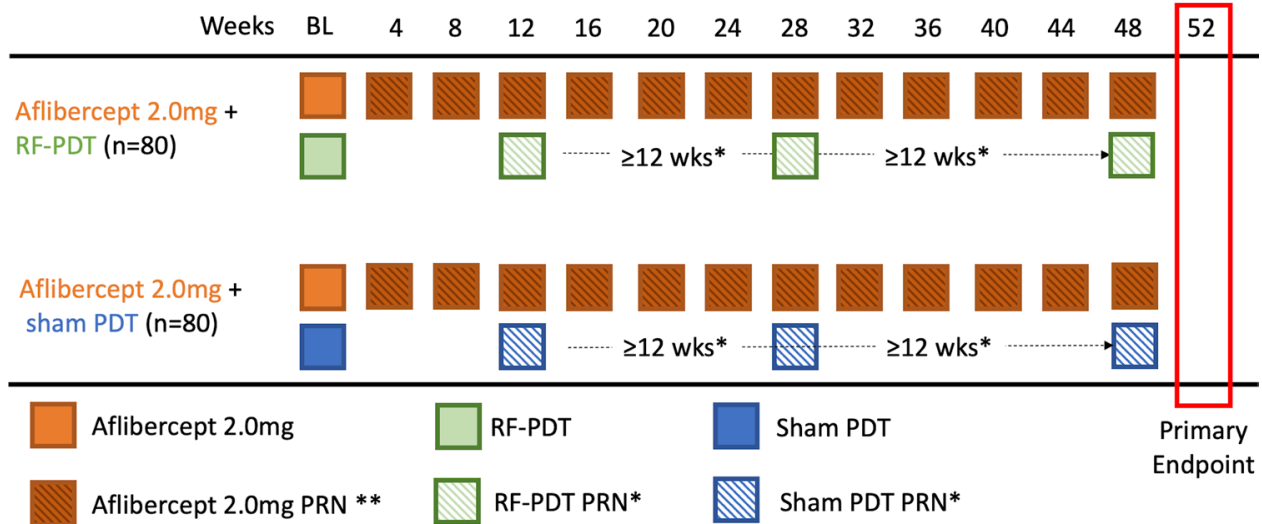

*RF-PDT/Sham PDT will be administered PRN as per protocol-specific retreatment criteria based on the presence of active polyp(s) on ICGA with a minimum interval between two PDT treatments of at least 12 weeks.

**Aflibercept administered PRN as per protocol-specific retreatment criteria if there was a BCVA loss or presence of

disease activity as seen on OCT. The interval between 2 aflibercept treatments must be least 28 days.

Figure 2 Visit schedules for each randomisation group. RF-PDT/Sham PDT will be administered PRN as per protocol-specific retreatment criteria based on the presence of active polyps on ICGA . Minimum interval between two PDT treatment will be at least 12 weeks. Aflibercept administered PRN as per protocol-specific retreatment criteria. The minimum interval between two aflibercept treatments will be at least 28 days. BCVA, best-corrected visual acuity; ICGA, indocyanine green angiography; OCT, optical coherence tomography; PDT, photodynamic therapy; PRN, pro re nata; RF-PDT, reduced-fluence photodynamic therapy.

infusion site will be disinfected prior to the infusion as per local standard.

This intravenous procedure will be done using the same cannula inserted into patient for FFA/ICG procedure. There will be no additional insertion of cannula. During the infusion, the delivery syringe and intravenous line should be wrapped in aluminium to mask the identity of treatment. The active RF-PDT/sham RF-PDT will be performed by an unmasked investigator enrolled in the study team.

\section{Active RF-PDT}

Verteporfin will be administered according to the current Visudyne package labelling. A dosage calculated at $6 \mathrm{mg} /$ $\mathrm{m}^{2}$ body surface area in a $30 \mathrm{~mL}$ solution will be infused intravenously over a $10 \mathrm{~min}$ period. Fifteen minutes after the start of the infusion (verteporfin), the study eye will be anaesthetised with topical anaesthetic and a contact lens will be used for the laser procedure. Laser light will be applied to the study eye for $83 \mathrm{~s}$ with the following parameters: light dose (reduced fluence) of $25 \mathrm{~J} / \mathrm{cm}^{2}$ and light wavelength of $689 \mathrm{~nm}$.

\section{Sham RF-PDT}

The sham infusion will be prepared with $5 \%$ dextrose in water solution for infusion. A $30 \mathrm{~mL}$ volume of this solution will be infused intravenously over a $10 \mathrm{~min}$ period to mimic the verteporfin infusion. Fifteen minutes after the start of the infusion (sham solution), the study eye will be anaesthetised with topical anaesthetic and a contact lens will be used for the laser procedure. A sham laser (ie, a true laser light will not be used) procedure that will mimic the procedure of the active RF-PDT will performed.

\section{Post-treatment care}

Patients who undergo RF-PDT will become temporarily photosensitive after the infusion. All patients who receive verteporfin RF-PDT/sham RF-PDT will be instructed to avoid direct sunlight for 48 hours.

\section{Treatment regimen adjustments}

If the study eye develops a treatment-related $\mathrm{AE}$ at any time during the study, treatment dose may be temporarily held and the reason for dose holding will be recorded in the case report file (CRF).

The treatment regimen will be adjusted based on the following criteria:

- Intraocular inflammation: may hold dose at the investigator's discretion, for example, if intraocular inflammation is $\geq 2+$ in the study eye. Treatment may resume when the inflammation has resolved.

- IOP: hold dose if IOP is $\geq 30 \mathrm{~mm} \mathrm{Hg}$ in the study eye. Treatment may resume when IOP is $\leq 30 \mathrm{~mm} \mathrm{Hg}$, either spontaneously or by treatment, as determined by the evaluating physician.

- New retinal break or retinal detachment: hold dose for the study eye. Treatment may resume after the retinal break/detachment had been successfully treated.

- Ocular and/or periocular infection: hold dose until the infection is resolved in both eyes.

The investigator may hold or discontinue study treatment for other safety reasons at his/her discretion.

\section{Randomisation and blinding}

Study participants will be randomised to two treatment groups using a ratio of 1:1 (combination group: aflibercept combined with RF-PDT, IVA monotherapy group: aflibercept monotherapy with sham RF-PDT) (figure 1), and randomisation will be performed using a blocked 


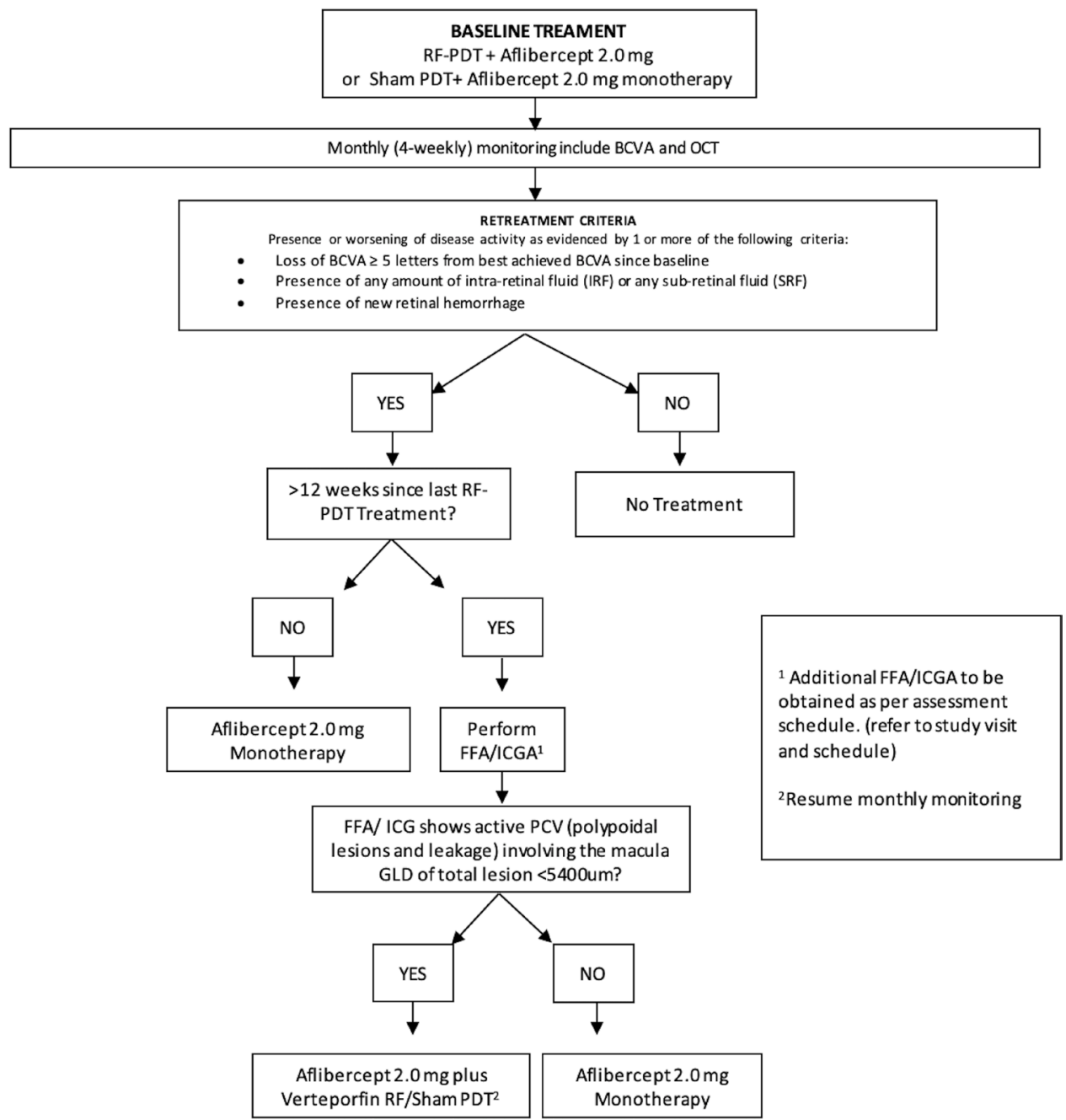

Figure 3 Retreatment criteria after baseline treatment. At each study visit, disease activity will be assessed by a masked investigator. Presence or worsening of the disease activity is considered if one or more of the following criteria are present: (1) loss of BCVA $\geq 5$ letters from best achieved BCVA since baseline, (2) presence of any amount of intraretinal fluid or any subretinal fluid, and (3) presence of new retinal haemorrhage. Depending on the duration since last the RF-PDT treatment, the patient will be treated either with monotherapy aflibercept ( $<12$ weeks since last RF-PDT) or will undergo FFA/ICG ( $>12$ weeks since the last RF-PDT). if angiographic analysis suggests polypoidal lesion involving macula with GLD $<5400$ um patient will undergo aflibercept monotherapy or aflibercept with RF-PDT/sham depending on the randomisation groups. If no activity is noted on angiographic analysis, only aflibercept monotherapy will be administered. BCVA, best-corrected visual acuity; GLD, greatest linear dimension; ICGA, indocyanine green angiography; OCT, optical coherence tomography; PDT, photodynamic therapy; RF, reduced-fluence; RF-PDT, reduced-fluence photodynamic therapy.

randomisation method. Each site will be randomised to a 1:1 ratio for each study treatment arm.

The site at the SNEC will be randomised in blocks of 20 and the remaining 18 participants will be randomised in blocks of 9 . For the TTSH site, 30 participants will be randomised in blocks of 10 . For the NUH site, 32 participants will be randomised in blocks of 20, with the remaining 12 participants randomised in blocks of 6 .

Blinding : Initial assessment and recruitment of the patient will be done by the masked coinvestigator and the masked research coordinators. The treatment procedure will be done by the unmasked coinvestigators. Both participants and masked team will be masked to treatment received.
Unblinding will be permissible only in circumstances involving SAE.

\section{Study completion}

All participants that have completed 52 weeks of treatment are considered to have completed the study successfully.

\section{Treatment schedule}

Treatment randomisation is summarised in figure 1.

All participant with macular PCV will be assessed at baseline and randomised to each study treatment arm (combination group: aflibercept combined with RF-PDT, IVA monotherapy group: Aflibercept monotherapy with sham RF-PDT) 
At the baseline visit, depending on the randomisation the combination group will receive IVA and RF-PDT while the monotherapy group with receive IVA+sham PDT.

After baseline visit, patients will be monitored at four weekly interval between weeks 8 and 48 .

At each study visit, disease activity will be assessed by masked investigator. If disease activity is present according to the prespecified treatment protocols (figure 3), patients will be treated according to randomisation arm. All clinical evaluations during study visits will be carried out by the masked investigator, while treatment administration will be undertaken by the unmasked investigator.

\section{Study medication}

Aflibercept (Eylea): study eyes will receive a dose of $2 \mathrm{mg}$ in $0.05 \mathrm{~mL}$ of aflibercept.

Verteporfin RF-PDT/sham RF-PDT: verteporfin will be administered according to the current Visudyne package labelling. A dosage calculated at $6 \mathrm{mg} / \mathrm{m}^{2}$ body surface area in a $30 \mathrm{~mL}$ solution will be infused intravenously over a 10 min period. Fifteen minutes after the start of the infusion (verteporfin), the study eye will be anaesthetised with topical anaesthetic and a contact lens will be used for the laser procedure. Laser light is applied to the study eye for $83 \mathrm{~s}$ with the parameters: light dose (reduced fluence) of $25 \mathrm{~J} / \mathrm{cm}^{2}$ and light wavelength of $689 \mathrm{~nm}$

\section{Dosage form, packaging and labelling}

Aflibercept (Eylea) is a solution for injection, clear, colourless to pale yellow, iso-osmotic solution, $\mathrm{pH}$ 6.2. following intravitreal injection of $2 \mathrm{mg}$ per eye of the mean Cmax of free aflibercept in the plasma was noted to be $0.02 \mathrm{mcg} / \mathrm{mL}$ (range: $0-0.054 \mathrm{mcg} / \mathrm{mL}$ ) and was attained in 1-3 days. The free aflibercept plasma concentration was undetectable 2 weeks after dosing.

Visudyne vial contains $15 \mathrm{mg}$ of verteporfin. After constitution, $1 \mathrm{~mL}$ contains $2 \mathrm{mg}$ of verteporfin. Five millilitres of reconstituted solution contains $15 \mathrm{mg}$ of verteporfin.

Sham RF-PDT consists of dextrose $5 \%$ solution followed by light application RF-PDT.

The sham infusion will be prepared with $5 \%$ dextrose in water solution for infusion. A $30 \mathrm{~mL}$ volume of this solution is infused intravenously over a $10 \mathrm{~min}$ period to mimic the verteporfin infusion.

\section{Storage and drug accountability}

Aflibercept (Eylea) will be stored in a refrigerator at $2^{\circ} \mathrm{C}-8^{\circ} \mathrm{C}$. It will be stored securely in the SERI Pharmacy, SNEC Building. It will be monitored by a 24-hour temperature monitoring system and report will be generated on a bioweekly basis. Alarms to any excursion will be triggered and the designated staff will be informed.

Visudyne will be stored in the original package in order to protect from light, at a room temperature not above $25^{\circ} \mathrm{C}$. It will be stored securely in the SERI Pharmacy cabinet with restricted access.
The label on each aflibercept/Visudyne/dextrose 5\% will include short study title, the name and country of origin of the manufacturer, batch number, trial number, expiry date, storage conditions, emergency contacts, subject number/initials, date of dispensed, visit/week and the words 'For clinical trial use only'.

\section{Concomitant treatment}

Any concomitant medications used by the subject from the date of enrolment until the end of the study except for routine medications given for ocular procedures required by the protocol, that is, fluoresceine, indocyanine, dilating drops and topical anaesthetics, should be recorded as concomitant medications, including the start and stop dates and indications.

Other non-anti-VEGF therapy or intravitreal corticosteroids in the study eye are not allowed.

If the fellow-eye needs treatment for wet AMD (including PCV), subjects can be treated in accordance to standard of care and continue in the study. All medications (prescription and over the counter), vitamin and mineral supplements, and/or herbs taken by the participant will be documented.

\section{Study completion and poststudy treatment}

Last visit will occur at week 52 ( \pm 7 days).

No special procedures will be carried out in addition to that stated in the schedule. Participants will not receive any treatment at the study completion visit.

Study participants will continue their routine follow-up with their regular physicians on completion of the final study. There will be no poststudy visits planned.

\section{Safety assessment}

Parameters

Safety parameters will include assessment of IOP, AEs and SAEs.

SAE and serious adverse reaction are defined as any untoward medical occurrence or effect that, at any dose result in death, is life threatening, requires hospitalisation or prolongation of existing hospitalisation, results in persistent or significant disability or incapacity, or is an important medical event.

\section{Adverse event}

AEs are defined as the following and do not need to be managed as serious: hospitalisation for routine treatment or monitoring of the studied indication not associated with any deterioration in condition, hospitalisation for elective or preplanned treatment for a pre-existing condition that is unrelated to the indication under study and has not worsened since the start of the study and treatment on an emergency outpatient basis for an event not fulfilling any of the definitions of a SAE given previously and not resulting in hospital admission.

Reporting and follow-up

Reporting of AEs involves the principal investigator submitting to the Centralised Institutional Review Board 
(CIRB) the SAE Reporting Form to CIRB within the stipulated timeframe. The principal investigator is responsible for informing the institution representative, the chairman medical board (when required by the institution for local SAE resulting in death), sponsor or regulatory bodies as required and appropriate.

\section{Reporting timeline to CIRB}

- Local unexpected SAE resulting in death that are related events should be reported immediatelywithin 24 hours of the principal investigator becoming aware of the event.

- Local unexpected, life-threatening SAEs that are related events should be reported as soon as possible but no later than seven calendar days after the principal investigator is aware of the event, followed by a full report within eight additional calendar days.

- Local unexpected, non-life-threatening SAEs that are related events should be reported no later than 15 calendar days after the principal investigator is aware of the event.

- An increase in the rate of occurrence of local expected SAEs that are related events, which is judged to be clinically important, should be reported within 15 calendar days after the principal investigator is aware of the event.

- Local unexpected AEs that are related events should be reported at least annually (together with the study status report for annual review).

- Non-local unexpected SAEs that are fatal or life threatening and related should be reported not later than 30 calendar days after the principal investigator is aware of the event. The review will be done on a monthly basis for standard aggregate AE safety data report.

The following standard aggregate AE safety data reports will be reviewed:

- Number and percentage of enrolled subjects reporting AEs by body system (ie, primary system organ class) and preferred term in descending frequency order.

- SAEs (treatment or procedure related and non-related)—cumulative.

- All AEs.

The following reports of additional study-specific data will be reviewed:

1. Targeted AEs.

- Intraocular inflammation/infection including AC inflammation, uveitis, vitritis, iritis, iridocyclitis, choroiditis, retinal vasculitis and endophthalmitis.

- Visual acuity reduced.

- IOP increase.

- Retinal tear.

- Retinal detachment.

- Vitreous haemorrhage.

- Retinal haemorrhage.

- Macular scar.

- Systemic VEGF inhibition.
2. Biomicroscopy/indirect ophthalmoscopy (by visit per subject).

$\geq 2$ grade increase in each separate parameter (corneal oedema, conjunctival hyperaemia, anterior chamber cells, anterior chamber flare, keratic precipitates, vitreous cells and vitreous flare/haze) and documented changes in vitreous, optic disc and retina from baseline.

3. BCVA of $\geq 15$ letters decrease from baseline at any visit (by visit per subject).

\section{Data governance and safety}

A Data and Safety Monitoring Committee (DSMC) will approve the protocol, template informed consent form and substantive amendments and provide independent monitoring of AEs. Cumulative AE data are semiannually tabulated for review by the DSMC. Following each DSMC data review, a summary will be made available for submission to the institutional review board. A list of specific AEs to be reported to the DSMC expeditiously will be compiled and included as part of the DSMC standard operating procedures.

\section{Data analysis}

Determination of sample size

Assuming a dropout 10\%, 80 subjects per treatment arm will be adequate to demonstrate non-inferiority of the combination arm (within a 5-letter non-inferiority margin, alpha $=0.025$ (one sided), power $=0.8$ ), assuming BCVA gain of 11.5 letters in the combination arm vs 10.7 letters in the monotherapy. Furthermore, we will be able to demonstrate superiority of combination over monotherapy arms with a 3-letter superiority difference assuming a BCVA gain of 13.7 letters in the combination arm vs 10.7 letters in the monotherapy (alpha $=0.025$ (one sided), power $=0.8$ ) and assuming a drop out $10 \%$ ). Furthermore, this same sample size will detect a difference in number of injections of 2 based on reducing from 7.3 in the monotherapy arm vs 5.2 in the combination arm with $>99 \%$ power (injection numbers are based on EVEREST II study data).

\section{Statistical methods}

For statistical purposes, baseline will be defined as the last available non-missing value collected just prior to the start of treatment in the study eye. For patients with screening assessments but who do not enter the treatment period, data will only be listed. For all patients, only one eye will be considered as the study eye, and only for this eye, efficacy analysis will be performed. Unless otherwise specified, all statistical tests will be two-sided with a 0.05 level of significance, and all CIs will be two-sided with $95 \%$ confidence level.

Categorical variables will be presented as the number and percentage of patients in each category. Continuous variables will be summarised using descriptive statistics (eg, n, mean, SD, median, minimum and maximum). Descriptive statistics will be provided for patient demographics and all baseline characteristics. Relevant medical 
history and current medical conditions will be tabulated by system organ class and preferred term of the MedDRA dictionary. Separate tables will be provided for ocular and non-ocular histories and conditions. Full analysis set (FAS) comprises all patients to whom treatment regimen has been assigned.

\section{Intent-to-treat}

Patients will be analysed according to the treatment regimen they are assigned to at randomisation. No data will be excluded from the FAS analyses because of protocol deviation. All efficacy evaluations will be carried out on the FAS.

Per protocol set (PPS) will consist of all patients in the FAS who followed the treatment regimen as randomised and completed week 24 without clinically significant protocol deviations.

Primary efficacy evaluation will be carried out on both the FAS and the PPS. The primary variable is the change in BCVA at week 52 compared with baseline. The primary analysis will be performed on the FAS using the LOCF approach for imputing missing data. The statistical testing will be carried out using paired t-test. The analysis will be repeated for the PPS using the same model.

Analysis of secondary endpoints will focus on the study eye only and will be based on the FAS. At all the time points assessed, each efficacy variable will be presented graphically and descriptive statistics will be provided based on absolute values and changes from baseline. For continuous and ordered categorical variables, changes from baseline will be compared between treatment groups using analysis of variance/analysis of covariance models/ t-test and stratified/unstratified Cochran-Mantel-Hansel tests. Stratification will follow the approach described for the primary analysis as applicable. Logistic regression will be used for analyses of binary endpoints.

\section{Primary and secondary variables}

\section{Primary variables}

Change in BCVA baseline to week 52 : BCVA at week 52 minus BCVA at baseline, loss of $\geq 5$ letters from BCVA since baseline.

\section{Secondary variables}

- Final BCVA at week 52.

- Proportion of eyes with gain of $\geq 5,10,15 \log M A R$ letters at week 52.

- Proportion of eyes with loss of $\geq 5,10,15 \log$ MAR letters at week 52.

- Proportion of eyes with 70 or more logMAR letters at week 52.

- Proportion of eyes with PL closure at weeks 12 and 52 assessed by ICGA.

- Proportion of eyes with presence of intraretinal and subretinal fluid at weeks 12 and 52 as evidenced by OCT.

- Mean number of intravitreal injections of aflibercept and RF-PDT.
- Frequency and severity of ocular and non-ocular AEs over 52 weeks.

- Evaluate the influence of anatomical imaging predictors such as CVI, CVH and sub-RPE hyper-reflective ring at weeks 12 and 52 between treatment groups (assessed by multimodal imaging).

- Longitudinal changes in OCTA between the groups.

\section{Reporting study deviations from the planned statistical analysis}

Deviations from the planned statistical analysis will be reported in the final study report.

\section{Safety analysis}

Only treatment-emergent AEs will be considered. The number and percentage of subjects reporting AEs will be reported.

\section{Access to source data/documents}

CRFs will be reviewed with the investigators study team before study initiation and baseline visit. During the study, the following parameters will be ascertained: (1) completeness of the records, (2) accuracy of entries, (3) adherence to the protocol and good clinical practices, (4) progress of the enrolment, and (5) storage, dispensing and accounting of the study medications source documentations will be available for monitoring and audit for compliance with clinical protocol. Monitoring standards require verification of presence of informed consent, adherence to the inclusion/exclusion criteria, report of SAEs and recording of data that will be used for efficacy and safety variables. Source documentation should not contain any participant identifiers.

\section{Ethics and dissemination}

This study will be conducted in accordance with the ethical principles that have their origin in the Declaration of Helsinki and that are consistent with the Good Clinical Practice and the applicable regulatory requirements.

This final Clinical Trial Protocol, including the final version of the participant information sheet and consent form, must be approved in writing by the CIRB and regulatory approval from Health Sciences Authority (HSA), prior to enrolment of any patient into the study.

\section{Regulatory and ethical compliance}

The investigator(s) and institution(s) will permit and facilitate all study-related monitoring audit(s) and regulatory review(s) and inspection(s), providing direct access to source data/documents.

\section{Informed consent procedures}

Potential eligibility will be assessed as part of a routinecare examination. Prior to completing any procedures or collecting any data that are not part of usual care, written informed consent will be obtained. For potential study participants who are considered potentially eligible for the study based on a routine-care exam, the study 
protocol will be discussed with the potential study participant by a study investigator.

The potential study participant will be given the Informed Consent Form to read. Potential study participants will be encouraged to discuss the study with family members and their personal physician(s) before deciding whether to participate in the study.

\section{Responsibilities of the investigator}

The principle investigator is responsible for informing the CIRB and HSA of any amendments to the protocol or other study-related documents, as per local requirement

\section{Data handling and record keeping}

Research data will be anonymised as soon as possible, and both identification key and the deidentified data will be stored in separate folders in the institution accesscontrolled shared folders. Case files will be kept under lock and key, with restricted access to the key, as defined within the study delegation log. The study data will be stored for at least 15 years and then destroyed or deleted.

\section{Patient and public involvement}

Patients are not invited to comment on the study design and are not consulted to develop patient-relevant outcomes or interpret results. Patients are not invited to contribute to the writing or editing of this document for reliability or accuracy.

\section{Financing and insurance}

Financial support is provided by National Medical Research Council Singapore Open Fund Large Collaborative Grant and covered under national clinical trial insurance policy.

\section{Publication policy}

On study completion and finalisation of the study report, the results of this trail will be submitted for publication in accordance to institutional publication policy of Singapore Eye Research Institute/SNEC

\section{Protocol adherence}

Investigators ascertain that they will apply due diligence to adhere to the study protocol and avoid protocol deviations

\section{Protocol amendments}

Change and addition to the protocol can only be made in written protocol amendment that must be approved by the CIRB. Amendments will require informed consent forms and/or other study-related material revision. In case of revision of informed consent form, all subjects enrolled in the study must sign the approved revised informed consent form.

\section{DISCUSSION}

Our study is aimed to ascertain the effectiveness of aflibercept monotherapy or in combination with RF-PDT using a PRN (pro re nata) treatment regimen. Prior reports such as The EVEREST II and PLANET studies have demonstrated that both anti-VEGF monotherapy and combination of anti-VEGF with PDT can achieve positive visual outcomes in eyes with PCV. ${ }^{14-17}$ In the EVEREST II study, the combination (IVR+PDT) group achieved superior visual gain compared with the monotherapy (IVR) group (+7 vs +5 letters gain) with on average two less IVR injections over 1 year. ${ }^{14}{ }^{15}$ In the PLANET study, both monotherapy arm (IVA) and IVA with rescue PDT arm achieved $>10$ letters improvement at 1 year and no benefit from rescue PDT was demonstrated. ${ }^{16}{ }^{17}$ Almost $78 \%$ of eyes achieved a fluid-free retina after the initial three loading doses of IVA.

Our study is also aimed to assess the importance of $\mathrm{PL}$ closure as a secondary endpoint for our study. PL closure is thought to be important for reducing the risk of longterm recurrence and massive submacular haemorrhage. ${ }^{18}$ We have defined PL closure as no PL detected on ICGA alone, which will be determined by our reading centre (SORC). Both the PLANET and EVEREST II studies have evaluated a similar anatomical end point defined as complete PL regression in the EVEREST II study and complete closure rates of PL in the PLANET study. Both these anatomical endpoints are defined as no PL lesion seen on ICGA. The EVEREST II study showed a significantly higher polyp regression in the combination arm compared with IVR monotherapy $(69.7 \%$ vs $33.8 \%){ }^{14}$ The proportion of patients with complete closure rate reported in the PLANET study was $38.9 \%$ for the IVA monotherapy and $44.8 \%$ for IVA with rescue PDT arm at week $52 .^{16}$

Two year results of PLANET reported that the favourable visual outcome of IVI monotherapy at year 1 was also maintained at second year and $<20 \%$ of the patients needed rescue PDT. However, patients requiring rescue PDT had significantly lower visual acuity gain. Unfortunately, untill today, there are only few studies evaluating the specific imaging or clinical characteristics to identify this group of poor responders and the role and timing of PDT and whether combination of IVA with PDT at baseline may achieve favourable visual and anatomical outcomes compared with IVA monotherapy. This study is aimed to address this question and will also evaluate if combination of IVA and PDT may lead to a reduction in the number of retreatments using an as-needed anti VEGF retreatment protocol.

Another novel aspect of this protocol is the use of RF-PDT as opposed to full fluence PDT used in EVEREST II trial. There have been concerns regarding full-fluence PDT, which include retinal haemorrhage post-treatment, choriocapillaris non-perfusion and damage to the retinal pigment epithelium. ${ }^{24-28}$ These concerns are particularly relevant in eyes which require repeated PDT treatments and large treatment spots. ${ }^{29}$ RF-PDT has been proposed to have better safety profile in terms of less RPE and choriocapillaris damage,${ }^{19} 20$ and few studies have shown promising results in retrospective case series with comparable 
visual improvements and polyp regression rates. ${ }^{20}{ }^{29}{ }^{30} \mathrm{~A}$ recent case-control review comparing reduced and standard fluence PDT in 38 patients with macular PCV showed a comparable visual gain and anatomical outcome with a polyp closure rate of $77.8 \%$ in the RF-PDT group. ${ }^{30}$ Although the effects of RF-PDT have been studied in context of PCV, the evidence comes from small sampled cases series and this study will give us the opportunity to evaluate the effect of RF-PDT more formally in a clinical trial setting.

Non- monthly treatment regimen as defined by disease activity is currently practiced by most physicians in the treatment of nAMD. These include mainly the treat and extend (TNE) regimen and PRN regimen. With continued focus on reducing the treatment burden and to improve the cost effectiveness for PCV, we wish to closely examine a PRN IVA regimen in this study. We believe that a PRN dosing regimen after an initial IVA injection at baseline with close monthly follow-up will allow us to identify the disease activity biomarkers that can predict the minimal effective IVA retreatment for PCV. Our proposed study is similar to the recently published clinical trial protocol for the Atlantic study that outlines a clinical trial aiming to evaluate the safety and efficacy of aflibercept in PCV either alone or in combination with PDT in the Caucasian population. ${ }^{31}$ However, unlike our study, this clinical trial aims to evaluate baseline full-fluence PDT and TNE regimen for retreatment.

The third aspect of this study is to evaluate imaging biomarkers that may predict treatment outcomes in PCV. Several retrospective clinical series have reported poorer response to IVR monotherapy in eyes with thicker choroid, and better visual outcome to PDT in eyes which exhibit $\mathrm{CVH}^{32}{ }^{33}$ A recent post hoc analysis from the EVEREST II study showed that a smaller polyp area at baseline was associated with better visual outcome with IVR monotherapy. ${ }^{34}$ Our study protocol will incorporate multimodal imaging in every study visit. We will evaluate imaging features specific to PCV, including PL and branching vascular network size on ICGA and OCT-based features such as sharp-peaked Pigment epithelium detachment (PED) and sub-RPE ring. ${ }^{356}$ In addition, we will also evaluate choroidal parameters such as choroidal thickness, CVH and CVI. Variations in choroidal characteristics may reflect a different predominant pathogenic process which in turn may explain the heterogeneity in treatment responses. ${ }^{22} 23323337-41$ We will also explore the use of newer imaging modality such as OCTA, which has the potential to delineate choroidal neovascularisation and PCV lesion components. These parameters will be tracked non-invasively with OCTA longitudinally. Changes in lesion characteristics on OCTA, such as increase in size and branching complexity may also be early indicators of disease reactivation. ${ }^{364-45}$ To date, there has been limited experience with OCTA due to various challenges like poor intervisit registration, artefacts and segmentation inaccuracy, which make quantitative analysis difficult. ${ }^{46}$ The OCTA data collected for this study will help to fill this important gap.
In summary, this protocol will address the clinical impact of combining IVA with RF-PDT and assess novel, PCV-specific imaging biomarkers. Together these results will fill important gaps in the current understanding of the management of PCV.

\section{Author affiliations}

${ }^{1}$ Singapore Eye Research Institute, Singapore

${ }^{2}$ Medical Retina, Singapore National Eye Centre, Singapore

${ }^{3}$ Department of Ophthalmology, Tan Tock Seng Hospital, Singapore

${ }^{4}$ Department of Ophthalmology, National University Hospital, Singapore

Contributors Study conception and design and grant holder: GCMG. Conception of the study: KYCT. Initiation of the study design and implementation: GCMG, KYCT, CT and CC. Provided statistical expertise in clinical trial design: JJ, CHV and KYCT. Institutional review board approval: GCMG, KW and KYCT. Manuscript drafting: CHV, GCMG and KYCT. Critical revision and refinement: TYW, AT, BF and SS. Refinement of the study protocol and approval of the final manuscript: all authors.

Funding Financial support is provided by the National Medical Research Council Singapore open fund large collaborative grant (NMRC/LCG/004/2018). Trial sponsor: Singapore Eye Research Institute/Singapore National Eye Centre (reference: SERI Ref. No. R1735/58/2020). Address: 11 Third Hospital Avenue, Singapore 168751, Singapore.

Competing interests None declared.

Patient consent for publication Not required.

Ethics approval This study involves human participants and was approved by an ethics committee or institutional board.

Provenance and peer review Not commissioned; externally peer reviewed.

Supplemental material This content has been supplied by the author(s). It has not been vetted by BMJ Publishing Group Limited (BMJ) and may not have been peer-reviewed. Any opinions or recommendations discussed are solely those of the author(s) and are not endorsed by BMJ. BMJ disclaims all liability and responsibility arising from any reliance placed on the content. Where the content includes any translated material, BMJ does not warrant the accuracy and reliability of the translations (including but not limited to local regulations, clinical guidelines, terminology, drug names and drug dosages), and is not responsible for any error and/or omissions arising from translation and adaptation or otherwise.

Open access This is an open access article distributed in accordance with the Creative Commons Attribution Non Commercial (CC BY-NC 4.0) license, which permits others to distribute, remix, adapt, build upon this work non-commercially, and license their derivative works on different terms, provided the original work is properly cited, appropriate credit is given, any changes made indicated, and the use is non-commercial. See: http://creativecommons.org/licenses/by-nc/4.0/.

ORCID iDs

Chui Ming Gemmy Cheung http://orcid.org/0000-0003-3358-3516

Tien Yin Wong http://orcid.org/0000-0002-8448-1264

Kelvin Yi Chong Teo http://orcid.org/0000-0002-6197-8681

\section{REFERENCES}

1 Yannuzzi LA, Wong DW, Sforzolini BS, et al. Polypoidal choroidal vasculopathy and neovascularized age-related macular degeneration. Arch Ophthalmol 1999;117:1503-10.

2 Lafaut BA, Leys AM, Snyers B, et al. Polypoidal choroidal vasculopathy in Caucasians. Graefes Arch Clin Exp Ophthalmol 2000;238:752-9.

3 Wong CW, Yanagi Y, Lee W-K, et al. Age-related macular degeneration and polypoidal choroidal vasculopathy in Asians. Prog Retin Eye Res 2016;53:107-39.

4 Balaratnasingam C, Lee W-K, Koizumi H, et al. Polypoidal choroidal vasculopathy: a distinct disease or manifestation of many? Retina 2016;36:1-8.

5 Cheung CMG, Lai TYY, Ruamviboonsuk P, et al. Polypoidal choroidal vasculopathy: definition, pathogenesis, diagnosis, and management. Ophthalmology 2018;125:708-24.

6 Rosenfeld PJ, Brown DM, Heier JS, et al. Ranibizumab for neovascular age-related macular degeneration. $N$ Engl $J$ Med 2006;355:1419-31. 
7 Brown DM, Kaiser PK, Michels M, et al. Ranibizumab versus verteporfin for neovascular age-related macular degeneration. $N$ Engl $J$ Med 2006;355:1432-44.

8 Heier JS, Brown DM, Chong V, et al. Intravitreal aflibercept (VEGF trap-eye) in wet age-related macular degeneration. Ophthalmology 2012;119:2537-48.

9 CATT Research Group, Martin DF, Maguire MG, et al. Ranibizumab and bevacizumab for neovascular age-related macular degeneration. N Engl J Med 2011;364:1897-908.

10 Lee WK, Kim KS, Kim W, et al. Responses to photodynamic therapy in patients with polypoidal choroidal vasculopathy consisting of polyps resembling grape clusters. Am J Ophthalmol 2012:154:355-65.

11 Yang S, Zhao J, Sun X. Resistance to anti-VEGF therapy in neovascular age-related macular degeneration: a comprehensive review. Drug Des Devel Ther 2016;10:1857-67.

12 Wong CW, Wong TY, Cheung CMG. Polypoidal choroidal vasculopathy in Asians. J Clin Med 2015;4:782-821.

13 Kokame GT, deCarlo TE, Kaneko KN, et al. Anti-Vascular endothelial growth factor resistance in exudative macular degeneration and polypoidal choroidal vasculopathy. Ophthalmol Retina 2019;3:744-52.

14 Koh A, Lai TYY, Takahashi K, et al. Efficacy and safety of ranibizumab with or without verteporfin photodynamic therapy for polypoidal choroidal vasculopathy: a randomized clinical trial. JAMA Ophthalmol 2017:135:1206-13.

15 Lim TH, Lai TYY, Takahashi K, et al. Comparison of ranibizumab with or without verteporfin photodynamic therapy for polypoidal choroidal vasculopathy: the Everest II randomized clinical trial. JAMA Ophthalmol 2020;138:935-42.

16 Lee WK, lida T, Ogura Y, et al. Efficacy and safety of intravitreal aflibercept for polypoidal choroidal vasculopathy in the planet study: a randomized clinical trial. JAMA Ophthalmol 2018;136:786-93.

17 Wong TY, Ogura Y, Lee WK, et al. Efficacy and safety of intravitrea aflibercept for polypoidal choroidal vasculopathy: two-year results of the aflibercept in polypoidal choroidal vasculopathy study. $A m \mathrm{~J}$ Ophthalmol 2019;204:80-9.

18 Cho JH, Ryoo N-K, Cho KH, et al. Incidence rate of massive submacular hemorrhage and its risk factors in polypoidal choroidal vasculopathy. Am J Ophthalmol 2016;169:79-88.

19 Yamashita A, Shiraga F, Shiragami C, et al. One-year results of reduced-fluence photodynamic therapy for polypoidal choroidal vasculopathy. Am J Ophthalmol 2010;149:465-71.

20 Yamashita A, Shiraga F, Shiragami C, et al. Two-year results of reduced-fluence photodynamic therapy for polypoidal choroidal vasculopathy. Am J Ophthalmol 2013;155:96-102.

21 Jirarattanasopa P, Ooto S, Tsujikawa A, et al. Assessment of macular choroidal thickness by optical coherence tomography and angiographic changes in central serous chorioretinopathy. Ophthalmology 2012:119:1666-78.

22 Koizumi H, Yamagishi T, Yamazaki T, et al. Relationship between clinical characteristics of polypoidal choroidal vasculopathy and choroidal vascular hyperpermeability. Am J Ophthalmol 2013;155:305-13.

23 Wei X, Ting DSW, Ng WY, et al. Choroidal vascularity index: a novel optical coherence tomography based parameter in patients with exudative age-related macular degeneration. Retina 2017;37:1120-5.

24 Cho JH, Park YJ, Cho SC, et al. Posttreatment polyp regression and risk of massive submacular hemorrhage in eyes with polypoidal choroidal vasculopathy. Retina 2020;40:468-76.

25 Nowak-Sliwinska P, van den Bergh $\mathrm{H}$, Sickenberg M, et al. Photodynamic therapy for polypoidal choroidal vasculopathy. Prog Retin Eye Res 2013;37:182-99.

26 Kang HM, Kim YM, Koh HJ. Five-year follow-up results of photodynamic therapy for polypoidal choroidal vasculopathy. $A m \mathrm{~J}$ Ophthalmol 2013;155:438-47.

27 Schmidt-Erfurth U, Laqua H, Schlötzer-Schrehard U, et al. Histopathological changes following photodynamic therapy in human eyes. Arch Ophthalmol 2002;120:835-44.

28 Recchia FM, Greenbaum S, Recchia CAC, et al. Self-reported acute decrease in visual acuity after photodynamic therapy for age-related macular degeneration. Retina 2006;26:1042-8.
29 Wong CW, Cheung CMG, Mathur R, et al. Three-Year results of polypoidal choroidal vasculopathy treated with photodynamic therapy: retrospective study and systematic review. Retina 2015;35:1577-93.

30 Ngo WK, Chee WK, Tan CS, et al. Comparing efficacy of reducedfluence and standard-fluence photodynamic therapy in the treatment of polypoidal choroidal vasculopathy. BMC Ophthalmol 2020;20:150.

31 Marques JP, Farinha C, Costa Miguel Ângelo, et al. Protocol for a randomised, double-masked, sham-controlled phase 4 study on the efficacy, safety and tolerability of intravitreal aflibercept monotherapy compared with aflibercept with adjunctive photodynamic therapy in polypoidal choroidal vasculopathy: the Atlantic study. BMJ Open 2017:7:e015785.

32 Cho HJ, Kim HS, Jang YS, et al. Effects of choroidal vascular hyperpermeability on anti-vascular endothelial growth factor treatment for polypoidal choroidal vasculopathy. Am J Ophthalmol 2013;156:1192-200.

33 Yanagi Y, Ting DSW, Ng WY, et al. Choroidal vascular hyperpermeability as a predictor of treatment response for polypoidal choroidal vasculopathy. Retina 2018;38:1509-17.

34 Cheung CMG, Tan CS, Patalauskaite R, et al. Ranibizumab with or without verteporfin photodynamic therapy for polypoidal choroidal vasculopathy: predictors of visual and anatomical response in the Everest II study. Retina 2021;41:387-92.

35 Cheung CMG, Lai TYY, Teo K, et al. Polypoidal choroidal vasculopathy: consensus Nomenclature and Non-Indocyanine green angiograph diagnostic criteria from the Asia-Pacific ocular imaging Society PCV Workgroup. Ophthalmology 2021:128:443-52

36 Teo KYC, Cheung GCM. New concepts in polypoidal choroidal vasculopathy imaging: a focus on optical coherence tomography and optical coherence tomography angiography. Asia Pac J Ophthalmol 2019. doi:10.22608/APO.201909. [Epub ahead of print: 27 Mar 2019].

$37 \mathrm{Kim} \mathrm{H}$, Lee SC, Kwon KY, et al. Subfoveal choroidal thickness as a predictor of treatment response to anti-vascular endothelial growth factor therapy for polypoidal choroidal vasculopathy. Graefes Arch Clin Exp Ophthalmol 2016;254:1497-503.

38 Sonoda S, Sakamoto T, Otsuka H, et al. Responsiveness of eyes with polypoidal choroidal vasculopathy with choroidal hyperpermeability to intravitreal ranibizumab. BMC Ophthalmol 2013;13:43.

39 Kim S-W, Oh J, Kwon S-S, et al. Comparison of choroidal thickness among patients with healthy eyes, early age-related maculopathy, neovascular age-related macular degeneration, central serous chorioretinopathy, and polypoidal choroidal vasculopathy. Retina 2011;31:1904-11.

40 Koizumi H, Yamagishi T, Yamazaki T, et al. Subfoveal choroidal thickness in typical age-related macular degeneration and polypoidal choroidal vasculopathy. Graefes Arch Clin Exp Ophthalmol 2011;249:1123-8.

41 Lee WK, Baek J, Dansingani KK, et al. Choroidal morphology in eyes with polypoidal choroidal vasculopathy and normal or subnormal subfoveal choroidal thickness. Retina 2016;36 Suppl 1:S73-82.

42 Cheung CMG, Yanagi Y, Mohla A, et al. Characterization and differentiation of polypoidal choroidal vasculopathy using swept source optical coherence tomography angiography. Retina 2017;37:1464-74.

43 Teo KYC, Yanagi Y, Lee SY, et al. Comparison of optical coherence tomography angiographic changes after anti-vascular endothelial growth factor therapy alone or in combination with photodynamic therapy in polypoidal choroidal vasculopathy. Retina 2018;38:1675-87.

44 Tomiyasu T, Nozaki M, Yoshida M, et al. Characteristics of polypoidal choroidal vasculopathy evaluated by optical coherence tomography angiography. Invest Ophthalmol Vis Sci 2016;57:OCT324-30.

$45 \mathrm{Kim}$ JY, Kwon OW, Oh HS, et al. Optical coherence tomography angiography in patients with polypoidal choroidal vasculopathy. Graefes Arch Clin Exp Ophthalmol 2016;254:1505-10.

46 Mendonça LSM, Perrott-Reynolds R, Schwartz R, et al. Deliberations of an international panel of experts on OCT angiography Nomenclature of neovascular age-related macular degeneration. Ophthalmology 2021;128:1109-12. 